\title{
Músculos artificiales y optomiografía aplicados a una mano robótica
}

\section{Artificial muscles and optomiography applied to a robotic hand}

\author{
Mariana Areiza ${ }^{1 *}$, José Mendoza ${ }^{l}$, Amilkar Saavedra ${ }^{l}$ José Serracin $^{l}$ \\ Licenciatura en Ingeniería de Sistemas y Computación, centro Regional de Chiriquí, Universidad Tecnológica de Panamá
}

Resumen Las prótesis actuales pueden llegar a costar miles de dólares. Aunque existan proyectos que desarrollen prótesis con impresoras 3d, la movilidad de estas se limita a abrir y cerrar la mano. En busca de aumentar la movilidad de una prótesis de mano sin aumentar el costo; esta investigación analiza el uso de músculos artificiales, creados a partir de hilo de pescar (nylon), desde una perspectiva experimental y matemática; para determinar las condiciones necesarias para el buen funcionamiento de dichos músculos en una prótesis. De igual manera, se propone el uso de sensores de pulso, de menor costo que los myowave, para distinguir el movimiento de los músculos biológicos y transmitirlo a los músculos artificiales.

Palabras clave Prótesis, Optomiografía, TPFA.

\begin{abstract}
Current prostheses can cost thousands of dollars. Although there are projects that develop prostheses with $3 \mathrm{~d}$ printers, the mobility of them is limited to open and close the hand. In search of increasing the mobility of a hand prosthesis without increasing the cost, this research analyzes the use of artificial muscles, created from fishing line (nylon), from an experimental and mathematical perspective; to determine the conditions necessary for the proper functioning of these muscles in a prosthesis. Similarly, it proposes the use of pulse sensors, cheaper than the myowave, to distinguish the movement of the biological muscles and transmit it to the artificial muscles.
\end{abstract}

Keywords Prostheses, Optomyography, TPFA.

* Corresponding authors: mariana.areiza @utp.ac.pa

\section{Introducción}

En la actualidad al menos 30 millones de personas en el mundo no poseen sus extremidades biológicas, especialmente manos. Estas carencias limitan considerablemente la independencia de un individuo por lo que se opta en adquirir una sustitución. Ciertamente las prótesis subsanan la pérdida de una extremidad gracias a sus fuertes materiales estructurales; sensores que miden la actividad eléctrica de los músculos del usuario, llamados myosensores; estos actuadores realizan el movimiento y su complejidad mecánica permite replicar los movimientos de una mano biológica, pero aumentan el costo de construcción y reducen la disponibilidad. En consecuencia de lo anterior, proyectos como E-NABLE o atomic lab reducen el costo de los materiales estructurales al modelar en programas $\mathrm{CAD}$ e imprimir en plástico las prótesis. Algunas de estas son mecánicas mientras otras poseen un myosensor y un servo motor como actuador; en ambos casos sus movimientos se limitan a abrir y cerrar la mano.

En esta investigación se explora aplicar la optomiografía y músculos artificiales para aumentar la movilidad de una mano, creada con una impresora 3D, con la menor cantidad de recurso monetario.

\section{Antecedentes}

Los robots actuales se mueven gracias al uso de motores rotacionales y adaptaciones mecánicas que transforman el movimiento rotativo en lineal. La precisión de los movimientos y los grados de libertad del robot dependen de la programación y cantidad de motores, lo que eleva considerablemente los costos de construcción.

La función principal de los músculos biológicos es dar movimiento al cuerpo al contraerse o relajarse. Esta característica es ideal para los movimientos finos y rápidos, por lo que es imperativo mimetizarla y aplicarla en robótica.

En robótica se denominan soft actuator a aquellos actuadores ligeros y elásticos. Estos tipos de actuadores representan una mejoría en la movilidad de robots por su fortaleza y posibilidad de miniaturización. Existen diversos tipos de soft actuator los cuales cambian su forma ante estímulos eléctricos, temperatura, luz o químicos. Entre los comunes están los polímeros electroactivos, EAP por sus siglas en inglés, aleaciones con memoria de forma (SMA), actuador de gel Bucky [1], actuador elastómero dieléctrico [2], actuador de compuestos metálicos de polímero [3], [4]. Otro tipo son los 
músculos neumáticos que utilizan el aire para cambiar de forma, son muy utilizados por la facilidad de fabricarlos y el grado de deformación de la estructura.

Los soft actuator más relevantes fueron los desarrollados en de la Universidad de Dallas [5]. En su investigación sugerían utilizar hilo de pescar retorcido y con forma de resorte para crear músculos artificiales; al que llamaron TwistedPolymeric-Fibre Actuators (TPFA). Este material mostraba la capacidad de expandir su diámetro mientras disminuía su longitud ante la presencia de calor. Para calentar las fibras de nylon, se cubren con metal por el que fluye una corriente eléctrica; esta provoca aumento de temperatura en el metal por causa del efecto Joule [6]. En consecuencia, el músculo TPFA puede ser controlado por un cambio de voltaje o corriente, igual que los motores DC.

Los TPFA pueden ser fabricados fácilmente por la disponibilidad comercial del nylon y los aportes de Semochkin, Aleksandr [7] quien desarrolló una máquina que permite la creación de estos tipos de músculos. Por estas características han sido aplicados en investigaciones como manos robóticas [8], [9], [10], dispositivos portátiles [11], y robots biomiméticos [12][13].

Para que los músculos TPFA reaccionen igual que músculos biológicos, pueden ser controlados a distancia gracias a mandos remotos o por lecturas de los músculos a través de la electromiografía (EMG) o mecanomiografía (MMG).

Una variante de la electromiografía se hace de forma superficial (s-EMG). Consiste en colocar unos electrodos superficiales para medir la corriente eléctrica generada por los músculos. Por otra parte, la tensión mecánica generada por los tendones es acompañado por el desplazamiento superficial de su respectivo músculo [14]. Estas técnicas requieren de sensores especiales de alto costo, sin embargo, permite capturar fielmente los movimientos del usuario y transmitirlos a los actuadores.

Las referencias [14], y [15] proponen una técnica no invasiva, que permite medir los cambios de forma en los músculos. Esta técnica, conocida como optomiografía, utiliza leds infrarrojos de banda corta Near Inflared Sensor (NIR).

La optomiografía es propuesta en el 2015 por Hamed Muhammed y Jammalamadaka Raghavendra en su investigación publicada bajo el nombre de Optomyography (OMG): A Novel Technique for the Detection of Muscle Surface Displacement Using Photoelectric Sensors [14]. En esta proponen que la OMG puede ser utilizada para las mismas funciones que MMG y s-EMG. Pero con la ventaja de reducir el ruido de fondo en las señales. Utiliza un sensor fotoeléctrico RPR-220 para medir los cambios superficiales de la piel que ocurren al mover un músculo biológico.
Para esta investigación se emplea el principio de la optomiografía de utilizar un led y un fotosensor para detectar el cambio de la absorción de la luz, cuando se contrae y relaja un músculo biológico; pero con la aplicación de fotopletismografía. La fotopletismografía es una técnica óptica simple para la obtención de dimensión de un órgano a través del cambio del volumen de la sangre, el cual provoca una alteración en la intensidad de la luz a través del órgano. Esta técnica es utilizada en sensores de pulso. El flujo de sangre equivale a la frecuencia cardiaca, debido a que la luz es absorbida por la sangre, por lo tanto, los pulsos de señales son directamente proporcionales a los pulsos del latido cardiaco.

\section{Principio de funcionamiento de músculos artificiales de nylon}

Los músculos artificiales de nylon son manufacturados a través de un sencillo proceso. Una fibra de nylon, llamado a partir de ahora precursor, es retorcida longitudinalmente varias veces. Esta torsión en las fibras las realinea, lo que a su vez añade al material una propiedad anisotrópica.

Según la dirección de las vueltas, las fibras pueden ser arregladas para crear un actuador lineal (homochiral) o de torsión (heterochiral) [16].

En la figura 1 se visualiza el arreglo de las fibras de nylon y se relaciona la dirección de estas con su respectivo nombre.

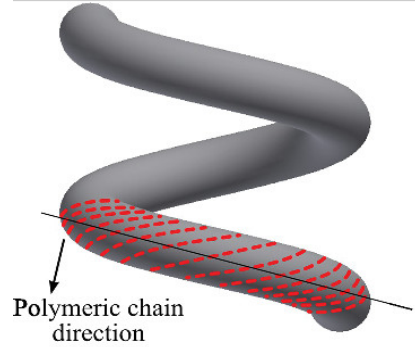

Homochiral

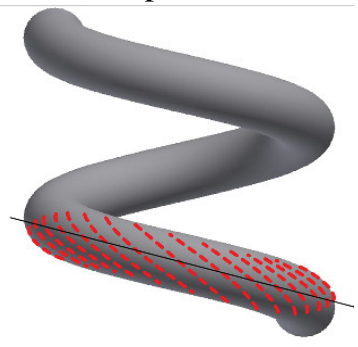

Heterochiral
Figura 1. Tipos de arreglo de fibra retorcida de nylon [5].

Generalmente los polímeros cristalinos, como el nylon, se endurecen al retorcer sus fibras debido las cadenas de polímero se orientan longitudinalmente a lo largo de las hélices lo que resalta las fibras. La gran elasticidad de entropía presente en las moléculas de enlace altamente estiradas provoca una expansión térmica negativa (NLTE) [17] en las fibras. En otras palabras, los polímeros cristalinos retorcidos se contraen en presencia de calor.

La respuesta térmica del TFPA depende principalmente de los siguientes tres factores.

- Diámetro del nylon precursor. 
- Índice de torsión, $\mathrm{C}$, definido como la relación entre el diámetro promedio de la bobina dividido por el diámetro de la fibra precursora [16].

- Ángulo de torsión de la fibra $\mathrm{a}_{\mathrm{c}}$, ver figura 2.

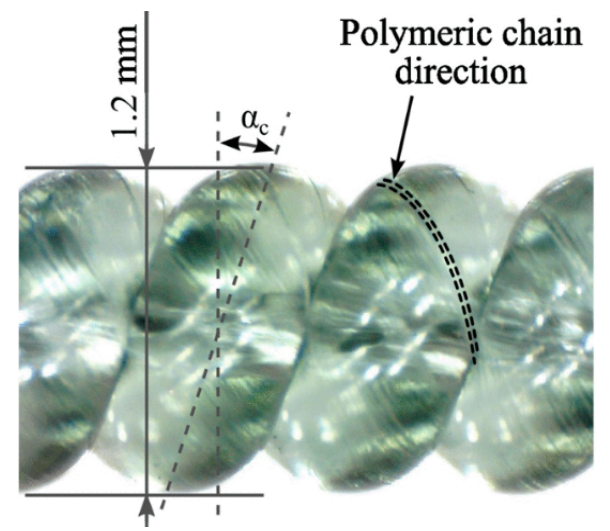

Figura 2. Lugar donde se ubica el ángulo a [5].

El ángulo de torsión se puede calcular con la fórmula (1) propuesta por [18] donde $\mathrm{D}$ es el diámetro del precursor en $\mathrm{mm}$ y $\mathrm{T}$ la cantidad de vueltas en vueltas $/ \mathrm{m}$ (turns $/ \mathrm{m}$ ).

$$
a_{c}=\tan ^{-1}(\pi \mathrm{DT})
$$

La reacción anisométrica de los músculos artificiales ante un estímulo térmico $\left({ }^{\Delta}\right.$ ) se puede calcular con la fórmula (2) donde D y Dl son los diámetros de la bobina, tomados a través de la línea central de la fibra antes y después del calentamiento respectivamente y $\alpha_{c}{ }_{c}^{\prime}$ es el cambio en el ángulo ac durante el estímulo térmico, como se describe en la ecuación mecánica de la investigación [19].

$$
\Delta T=\frac{\sin \left(\alpha_{\mathrm{c}}^{\prime}\right) \cos \left(\alpha_{\mathrm{c}}^{\prime}\right)}{\pi D^{\prime}}-\frac{\sin \left(\alpha_{\mathrm{c}}\right) \cos \left(\alpha_{\mathrm{c}}\right)}{\pi D}
$$

Para una bobina de $\mathrm{N}$ vueltas y longitud $\mathrm{L}$ hecha de una fibra de longitud 1 se puede describir con las fórmulas (3) y (4). Usando estas relaciones y suponiendo un cambio insignificante en la longitud de la fibra 1 se obtiene la fórmula (5). Es así como la fórmula (5) se puede determinar el cambio de torsión en un músculo de nylon colocar un peso; a partir del número de vueltas $\mathrm{N}$, es el cambio de la longitud del músculo al ser estirado $\Delta \mathrm{L}$ y la longitud inicial del precursor 1 .

$$
\begin{gathered}
\sin \left(\alpha_{c}\right)=\frac{L}{l} \\
\cos \left(\alpha_{c}\right)=\frac{\pi N D}{l}
\end{gathered}
$$

$$
\Delta T=\frac{N \Delta L}{l^{2}}
$$

En [20] se propone un modelo matemático para los TCPA que se expresa como una conexión en serie del modelo termo mecánico fórmula (6) y el modelo termoeléctrico (7):

$$
\begin{gathered}
m \frac{d^{2} y(t)}{d t^{2}}+d \frac{d y(t)}{d t}+k y(t)=c \Delta T(t), \\
C_{v} \frac{d \Delta T(t)}{d t}=-\alpha S_{c} \Delta T(t)+\frac{v^{2}(t)}{R}
\end{gathered}
$$

De las dos ecuaciones anteriores [21] las relaciona para obtener la ecuación diferencial (8).

$$
\frac{d^{3} y(t)}{d t^{3}}+a_{2} \frac{d^{2} y(t)}{d t^{2}}+a_{1} \frac{d y(t)}{d t}+a_{0} y(t)=b_{0} v^{2}(t)
$$

La ecuación diferencial (8) de tercer orden, describe el comportamiento de desplazamiento del resorte en tener presencia de calor, donde los coeficientes $\mathrm{a} 2=(\mathrm{dCv}+\mathrm{m \alpha Sc})$ $/ \mathrm{mCv}, \mathrm{a} 1=(\mathrm{kCv}+\mathrm{d} \alpha \mathrm{Sc}) / \mathrm{mCv} \mathrm{a} 0=\mathrm{k} \alpha \mathrm{Sc} / \mathrm{mCv}, \mathrm{y} \mathrm{b} 0=$ $\mathrm{c} / \mathrm{mRCv}$. Las variables y constantes d, Cv, m, Sc, k, V2(t), R y $\alpha$ corresponden al coeficiente de amortiguamiento, capacidad calorífica, masa, área de la superficie, constante de resorte, voltaje de entrada, resistencia eléctrica, coeficiente proporcional de una variación de temperatura a una fuerza generada y el coeficiente de transferencia de calor, respectivamente.

\section{Principio de funcionamiento de sensor pulso para optomiografía}

El sensor del pulso empleado en esta investigación es el sen-11574. Este sensor incluye un led verde (AM2520ZGC09), un fotosensor (APDS9008), un amplificador operacional y un conversor analógico digital (ADC0831). Utiliza la fotopletismografía de transmisión que mide el pulso cardiaco al calcular la reflexión de la luz del led verde con el fotosensor; este mismo principio puede utilizar para calcular si un músculo esta relajado o tenso. A medida que el músculo se contrae, la cantidad de luz reflejada incrementa, figura 3 y 4 , lo que provoca que la corriente en APDS9008 aumente y así mismo el voltaje.

La señal analógica producida por el fotosensor es amplificada y normalizada por amplificador operacional. La 
salida del amplificador operacional es conectada a un pin analógico en el Arduino.

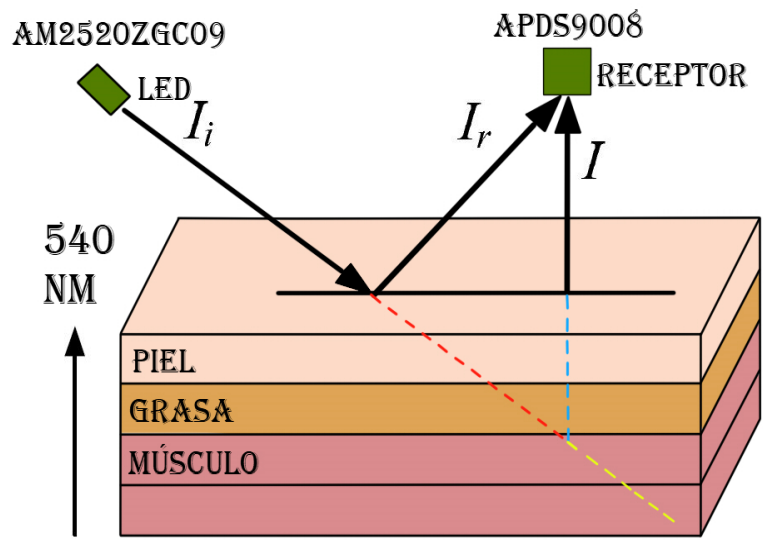

Figura 3. Músculos biológicos relajados. Fuente: elaboración propia.

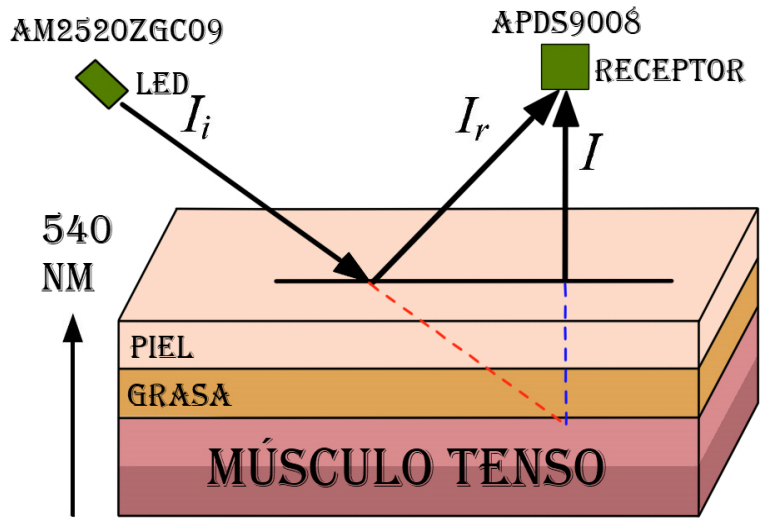

Figura 4. Músculos biológicos tensos. Fuente: elaboración propia.

\section{Métodos y materiales}

Para facilitar el análisis y desarrollo, la investigación se divide en actividades que se enuncian a continuación.

\subsection{Actividad 1: Crear músculos artificiales a partir de nylon}

Para crear los músculos artificiales de nylon se imprimen piezas en una impresora 3D y se arma la Artificial muscle twister (AMT), figura 5, diseñada y creada por Aleksandr N. Semochkin de la universidad Blagoveshchensk, Russia, para hacer músculos artificiales a partir de hilos de nylon y cobre como actuador[7]. Debido a que la varilla T8 que propone Semochkin no está disponible en Panamá y en busca de reducir costos, se remplaza por una M3 y se cambia el software de la máquina para que funcione con este tipo de varilla roscada.

El precursor utilizado fue el hilo de pescar Araty de $0.8 \mathrm{~m}$.

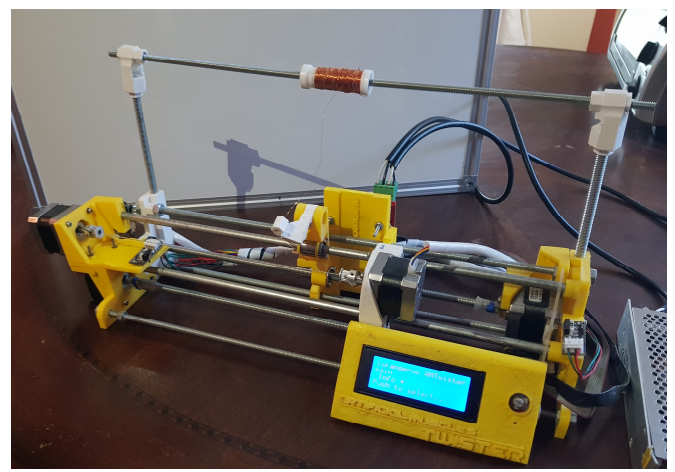

Figura 5. Artificial muscle twister. Fuente: elaboración propia.

Para crear un músculo artificial, a partir de nylon, con la AMT, se siguen los pasos enumerados a continuación:

- Stretch modo (figura 6) el motor SM3 gira, lo que provoca que el motor SM2 se aleje hasta que el hilo de nylon se tense.

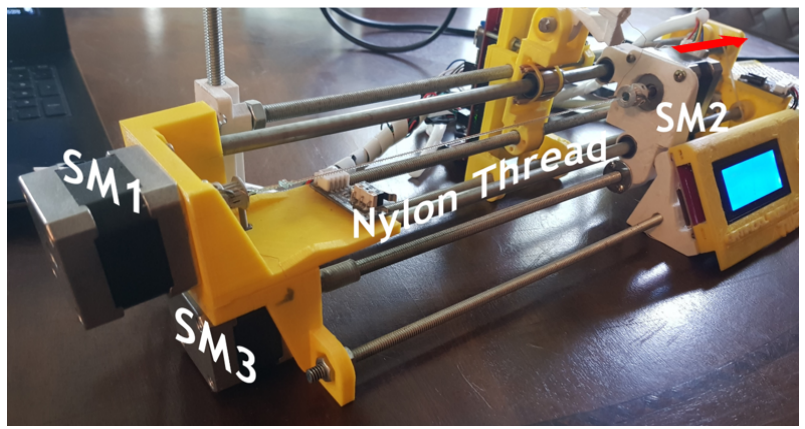

Figura 6. Artificial muscle twister Stretch modo. Fuente: elaboración propia.

- Pre-Coiling como se observa en la figura 5, el motor SM1 y SM2 giran en sentido opuesto. Esto provoca que el hilo de nylon se enrolle sobre sí mismo y cree el ángulo de torsión de la fibra que se muestra en la figura 7.

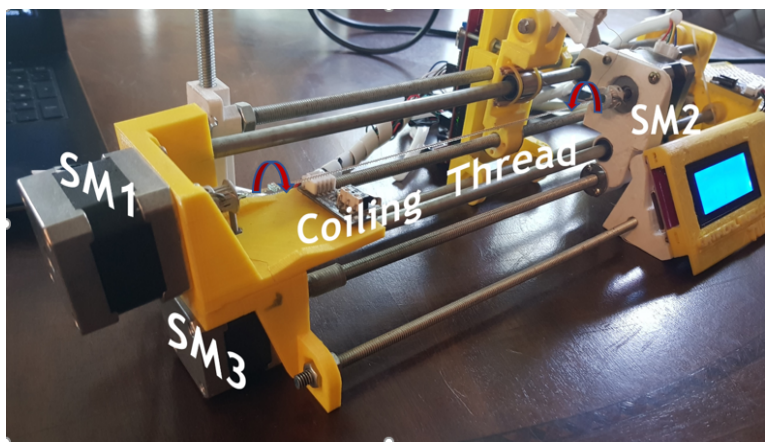

Figura 7. Artificial muscle twister Pre-Coiling. Fuente: elaboración propia. 
- Mandrel Coiling: El hilo de nylon rodeado con cobre se enrosca en un alambre dulce de aluminio (figura 8).

- Hornear: para que el hilo de nylon mantenga la forma de resorte se hornea a $65^{\circ} \mathrm{C}$ por 30 minutos.

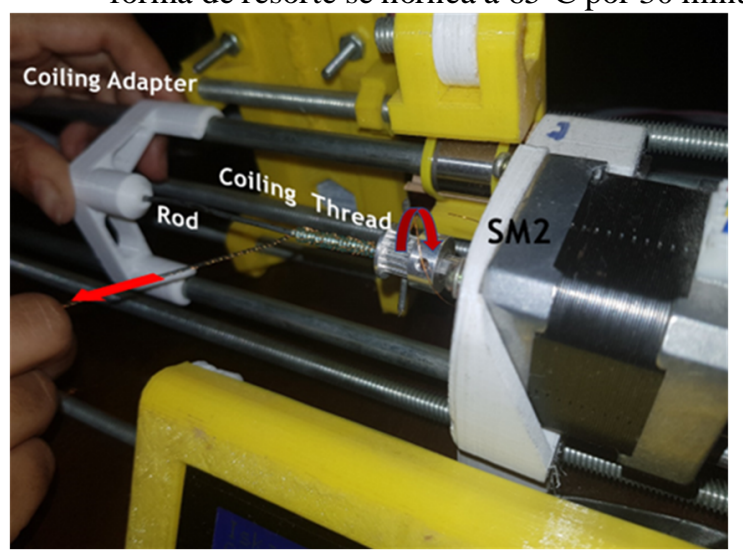

Figura 8. Artificial muscle twister etapa mandrel Coiling. Fuente: elaboración propia.

El diagrama de flujo presentado en la figura 9 corresponde a la actividad 1. En este se contemplan los pasos para desarrollar los músculos artificiales.

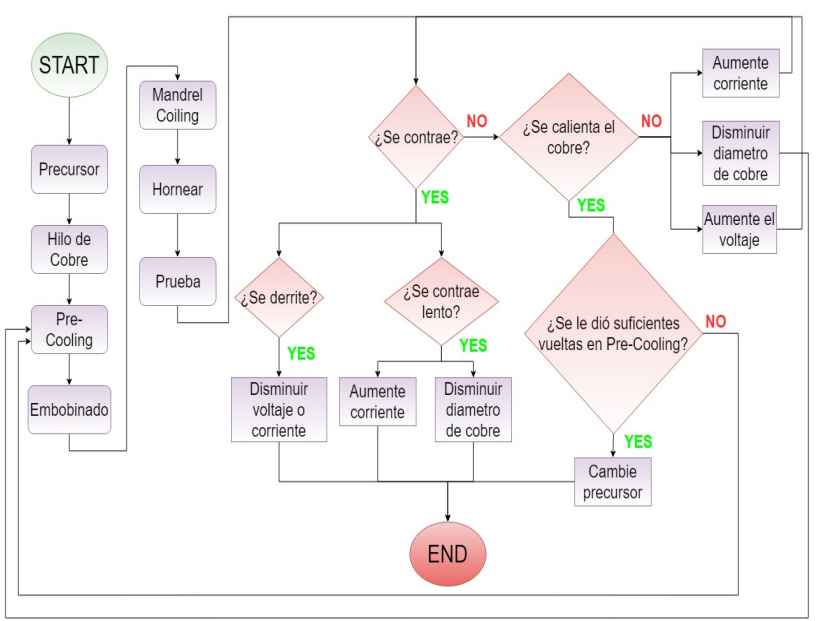

Figura 9. Diagrama de flujo de la etapa 1, músculos artificiales.

Con el circuito de la figura11 se analiza el comportamiento de los músculos artificiales antes creados. Para diferenciar los músculos se les nombra con letras de abecedario, se mide su resistencia, las distancia D1 corresponde al largo del musculo con un peso de $28 \mathrm{~g}$ sin aplicar el voltaje y la D2 corresponde cuando el voltaje es aplicado como se muestra en la figura10, también se mide el tiempo que le toma al musculo contraerse hasta su límite. Los datos anteriores se aplican para dos tipos de músculos; unos cuyo hilo de cobre es de $0.0068 \mathrm{~mm}$ y otro para 0.08 .

\section{COMPARACIÓN DE MÚSCULOS \\ CON Y SIN VOLTAJE}
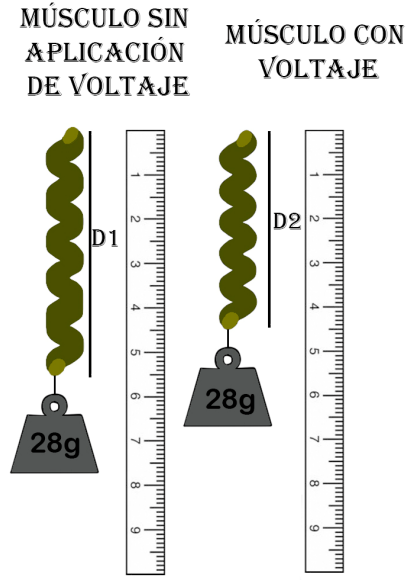

Figura 10. Medida de distancia D1 y D2.

El polímero electroactivo (TCPA) es activado mediante el efecto Joule y se controla variando el voltaje aplicado. El TCPA tiene como respuesta la relación entre la potencia aplicada y el efecto Joule.

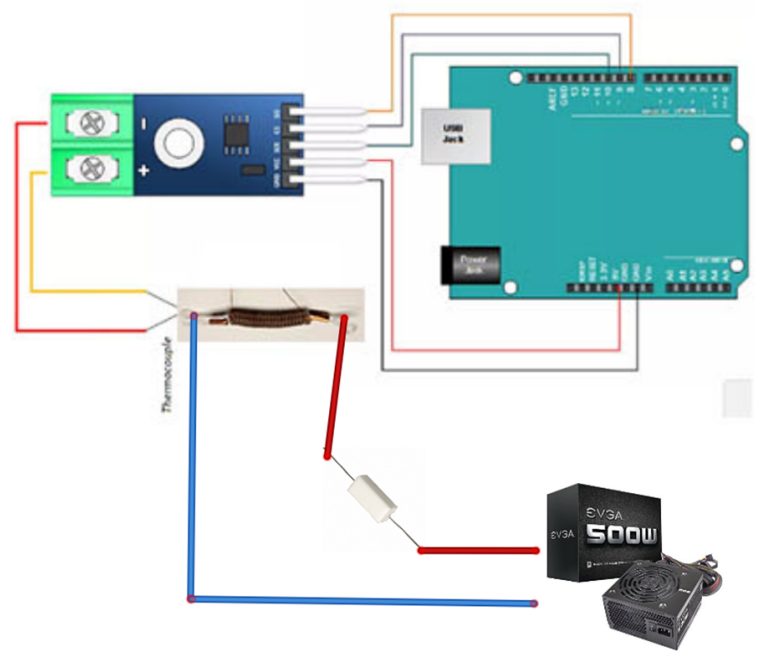

Figura 11. Circuito de prueba para músculos artificiales.

En busca de un modelo matemático describa el comportamiento de los músculos artificiales descritos en este artículo, se utiliza la ecuación diferencial (8). Las variables y coeficientes se obtienen de los datos experimentales se describen en el punto 6 de este artículo.

\subsection{Actividad 2: Experimentación de sensor sen-11574 para optomiografía}

Cuatro sensores de pulso sen-11574 se colocan sobre el flexor común profundo de los dedos. Según la posición donde se coloque se puede medir la tensión provocada al flexionar 
alguno de los dedos. Los sensores se disponen sobre el músculo como se muestra en la figura 12.

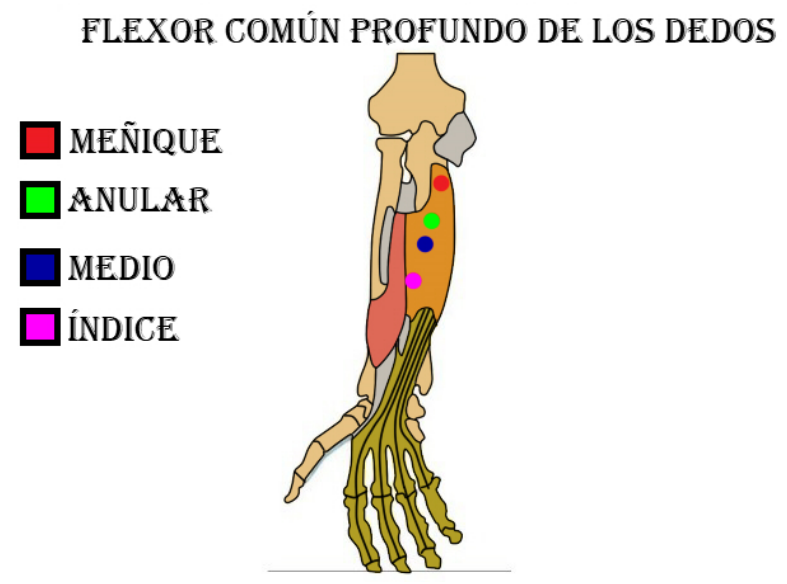

Figura 12. Posición de los sensores de pulso en el músculo flexor común profundo de los dedos.

Una vez colocados los sensores en el músculo, se conecta el sensor al Arduino para realizar una lectura analógica. La lectura de flexionar y relajar el musculo se presentan en la gráfica 2.

Para comparar las señales del sensor de pulso y un myosensor que utiliza electromiografía, se coloca en el músculo un myosensor sen-13723, luego se conecta al Arduino en un pin analógico y se registran las mediciones en la gráfica 3.

\subsection{Actividad 3: Aplicar músculos artificiales optomiografía a una mano hecha en impresora 3D}

Los músculos se disponen en la mano robótica como se muestra en la figura 14 y activan con un MOSFET IRFZ44N. Las conexiones entre los componentes y Arduino se realizan como se muestra en la figura 13.

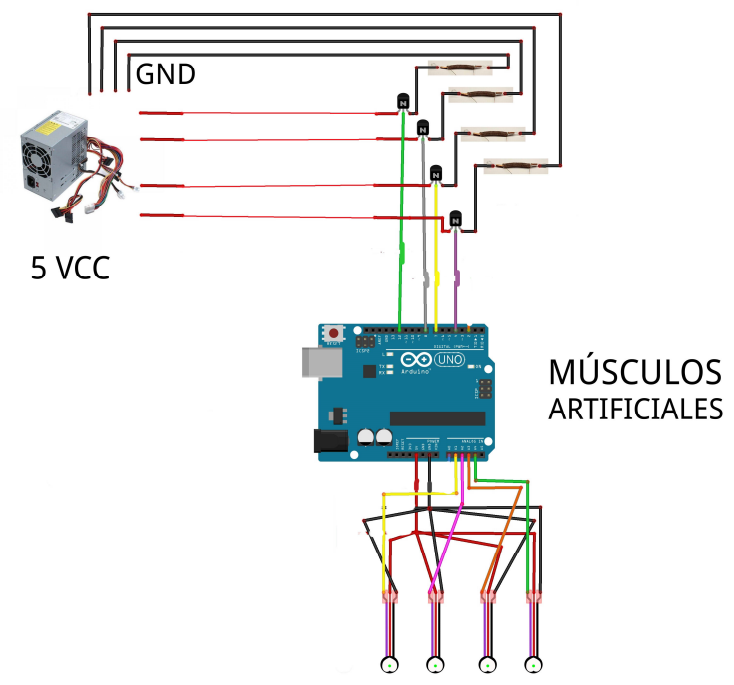

Figura 13. Circuito final del proyecto.

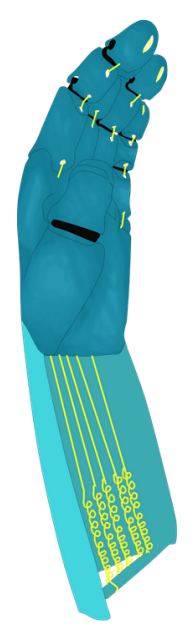

Figura 14. Disposición final de los músculos en la mano robótica.

\section{Resultados}

En esta sección se analizan los datos obtenidos por actividad.

\subsection{Actividad 1:}

La gráfica 1 representa el tiempo obtenido en las seis pruebas realizadas con diferentes músculos artificiales, dado que el grosor y la resistencia de cada músculo jugaron un valor importante en la obtención de estos datos, como se muestra en la tabla 1; se da a conocer los valores tales como: resistencia del músculo, distancia inicial y final recorrido luego de aplicarle 5V, temperatura obtenida al llegar al límite requerido, el grosor del cobre que está estimado en las pruebas antes mencionadas de $0.0063 \mathrm{~mm}$ y $0.08 \mathrm{~mm}$, respectivamente.

Para cada uno de los músculos se crea a partir de un precursor de largo de $27 \mathrm{~cm}$, en la etapa de precooiling, al precursor se le da 105 vueltas y el diámetro del precursor es de 0.8, es así como al aplicar la fórmula (1) el ángulo es:

$$
a_{c}=\tan ^{-1}(0.8 \times \pi \times 105)=89.78
$$

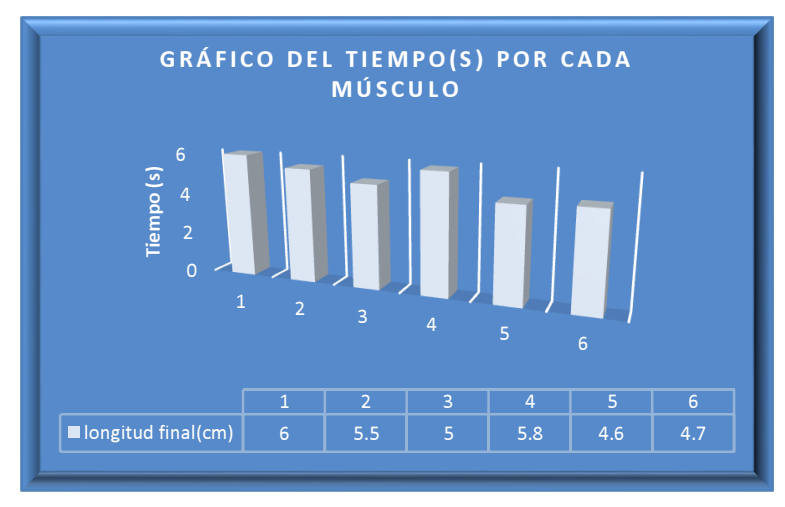

Gráfico 1. Tiempo obtenido con los seis músculos creados en esta investigación. 
Tabla 1. Valores de los músculos artificiales creados en este artículo

\begin{tabular}{|c|c|c|c|c|c|c|}
\hline nombre & $\begin{array}{c}\text { Resistencia } \\
\text { del músculo } \\
(\Omega)\end{array}$ & $\begin{array}{c}\text { D1 } \\
(\mathbf{c m})\end{array}$ & $\begin{array}{c}\text { D2 } \\
(\mathbf{c m})\end{array}$ & $\begin{array}{c}\text { Temperatura } \\
\left({ }^{\circ} \mathbf{C}\right)\end{array}$ & $\begin{array}{c}\text { Grosor } \\
\text { del alambre } \\
(\mathbf{m m})\end{array}$ & tiempo (S) \\
\hline A & 4.5 & 6.5 & 6 & 41 & 0.0063 & 25 \\
\hline B & 3 & 6.5 & 5.5 & 41.5 & 0.0063 & 24 \\
\hline C & 3.2 & 6 & 5 & 43 & 0.0063 & 22 \\
\hline D & 3.2 & 6 & 5.8 & 28 & 0.08 & 98 \\
\hline E & 2.7 & 5 & 4.6 & 33 & 0.08 & 96 \\
\hline F & 2.7 & 5 & 4.7 & 32 & 0.08 & 97 \\
\hline
\end{tabular}

Los datos experimentales obtenidos en la tabla 1 se remplazan en la ecuación diferencial (8) para obtener el modelo matemático que corresponde a los músculos TPFA creados a partir de un precursor de nylon poliamida 6 , de un diámetro de $0.8 \mathrm{~mm}$ y con un voltaje de $5 \mathrm{v}$.

En la siguiente tabla se describe los valores de cada variable y constante de la ecuación diferencial (8).

Tabla 2. Valores que corresponden a las variables para sustituir en ecuación diferencial (8)

\begin{tabular}{|l|l|}
\hline \multicolumn{1}{|c|}{ Variable/Constante } & Valor \\
\hline $\begin{array}{l}\text { Capacidad calorífica de la } \\
\text { poliamida 6 (Cv) }\end{array}$ & 0.38 \\
\hline $\begin{array}{l}\text { Coeficiente de transferencia de } \\
\text { calor de a poliamida 6( } \alpha)\end{array}$ & 0.25 \\
\hline Masa del objeto(m) & $0.222 \mathrm{~kg}$ \\
\hline Constante de resorte(k) & 62.16 \\
\hline $\begin{array}{l}\text { Coeficiente de } \\
\text { amortiguamiento(d) }\end{array}$ & 1.182 \\
\hline Voltaje de entrada(v) & $5 \mathrm{v}$ \\
\hline Área de la superficie $(\mathrm{Sc})$ & 0.01068 \\
\hline Resistencia eléctrica $(\mathrm{R})$ & $4.5 \Omega$ \\
\hline $\begin{array}{l}\text { Coeficiente proporcional de una } \\
\text { variación de temperatura a una } \\
\text { fuerza generada }\end{array}$ & $1.23 \times 10^{-3}$ \\
\hline
\end{tabular}

De los datos de la tabla 2 se obtiene los siguientes coeficientes $\mathrm{A} 1=280.03, \quad \mathrm{~A} 2=5.33, \mathrm{~A} 0=1.9673 \mathrm{y}$ $\mathrm{B} 0=3.24 \times 10-3$ sal sustituir en la ecuación diferencial (8) se obtiene $\mathrm{Y}(\mathrm{t})=0.558354 \mathrm{e}(-7 \times 10-3) \mathrm{t}+4.73 \times 10-4 \mathrm{e}(-2.66) \mathrm{t}$ sen $(16.52)+1.469 \times 10-3 \mathrm{e}(-2.66) \mathrm{t} \operatorname{Cos}(16.52) \mathrm{t}+0.041173$ que representa un modelo matemático que relaciona la contracción de un musculo TPFA con respecto al tiempo.

\subsection{Actividad 2:}

La grafica 1 refleja el cambio en el voltaje en el sensor de pulso al mover el dedo anular.

Para las gráficas 1 y 2 el eje "Y" corresponde a la lectura en milivoltios del puerto analógico del Arduino y " $\mathrm{X}$ " corresponde al tiempo en milisegundos.

En la gráfica 1 se muestra en azul la medida obtenida por la tarjeta Arduino con el sensor de pulso en el dedo medio. Los valores arriba de la línea roja corresponden a la flexión del musculo biológico y los de abajo corresponden a la relajación del musculo biológico.

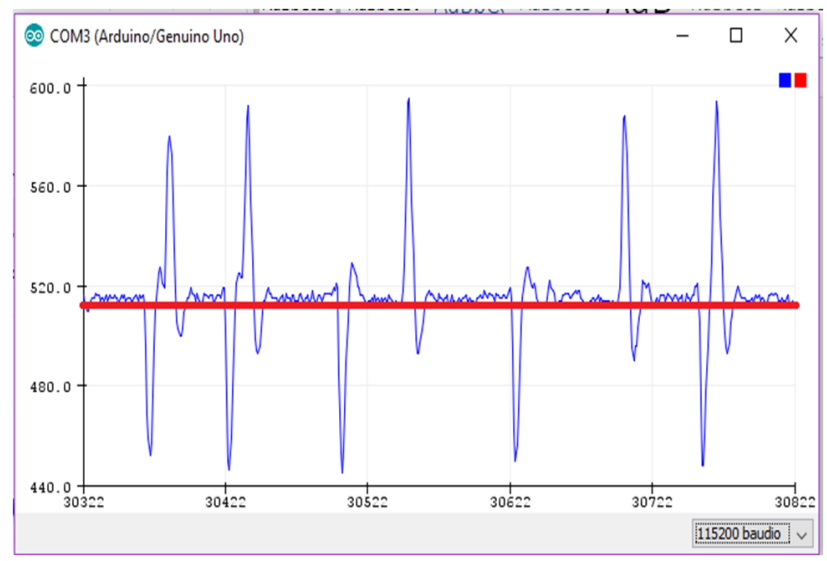

Gráfica 2. Medidas de contracción de músculo biológico con sensor de pulso.

La gráfica 2 se muestra en azul la medida obtenida por la tarjeta Arduino con el myosensor sen-13723 en colocado en el codo. El flanco de subida de la señal corresponde a la contracción del musculo y el flanco de bajada a la etapa de relación del musculo.

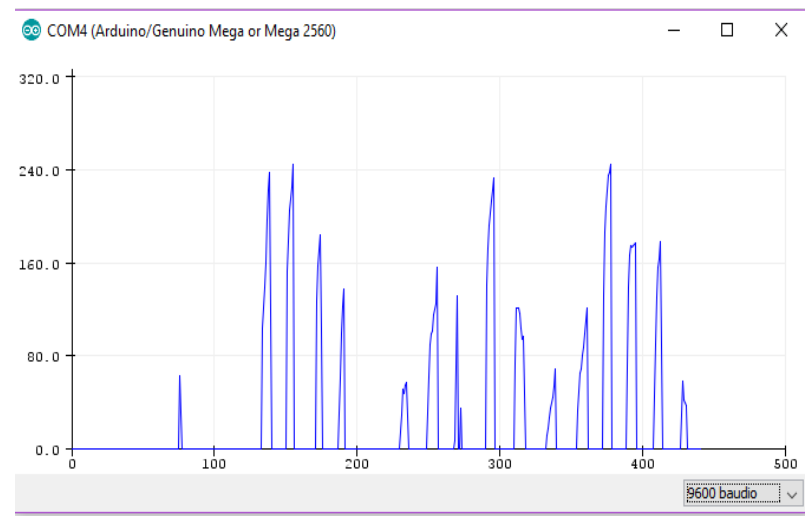

Gráfica 3. Medidas con myosensor.

\subsection{Actividad 3:}

En la figura 12 se observa el circuito final del proyecto, los MOSFET funcionan como interruptor para activar los músculos. 


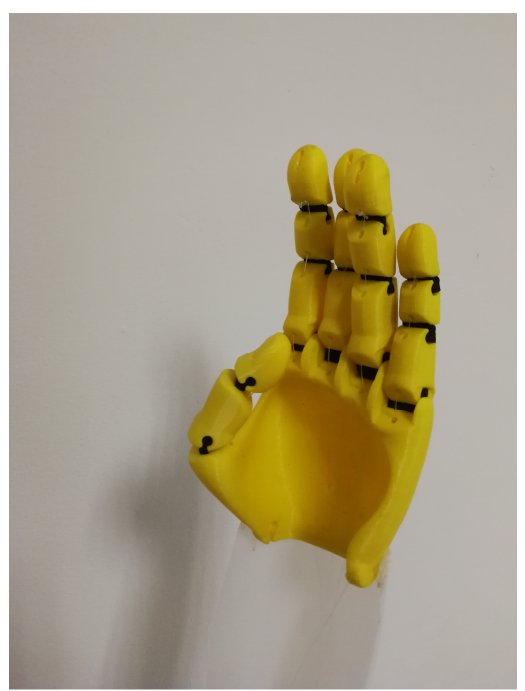

Figura 15. Mano hecha con impresora 3D utilizada en este proyecto.

En la figura 15 se observa la mano impresa en 3D utilizada en este proyecto.

Lo que se muestra en la figura 16 corresponde al movimiento del dedo meñique al aplicar uno de los músculos antes creados.

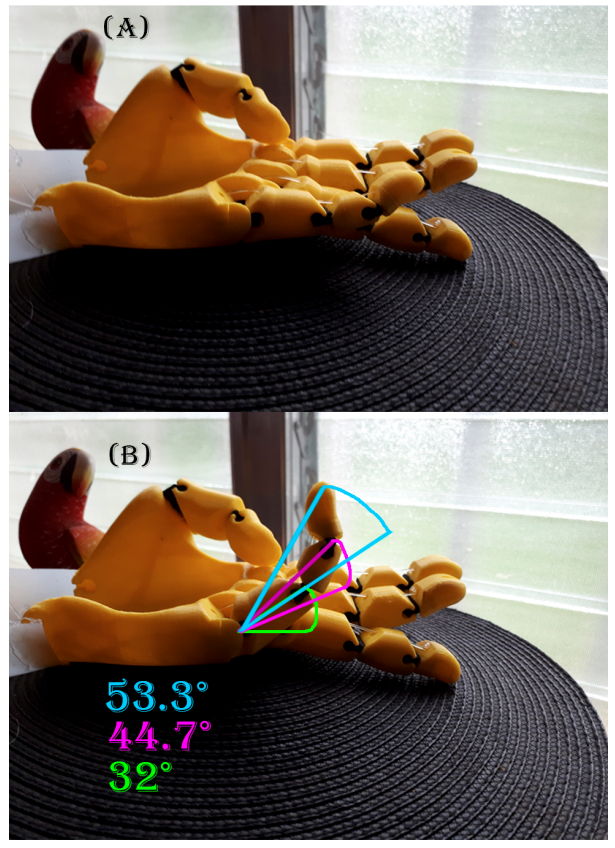

Figura 16. Mano hecha con impresora 3D empleando músculos artificiales en el dedo meñique.

\section{Discusión y conclusiones}

Este trabajo de investigación ofrece una alternativa más económica y sencilla para lectura de movimiento muscular que los métodos antes propuestos. En [2] el uso de un sencillo sensor infrarrojo y de un osciloscopio dificultaba la implementación de método, además el sensor rpr-220 es difícil de encontrar actualmente.

Para la obtención de estos datos se utilizó la nueva configuración de los músculos el cual según la gráfica 1 es más efectiva el uso de cable de cobre de $0.0063 \mathrm{~mm}$, ya que disminuye considerablemente el tiempo de ejecución de las pruebas realizadas con una pesa de $28 \mathrm{~g}$. Mientras que, con el cobre de $0.08 \mathrm{~mm}$ se representa una diferencia de $74.49 \%$ en la reducción del tiempo entre los cobres de diámetro $0.0063 \mathrm{~mm}$ y $0.08 \mathrm{~mm}$.

La gráfica 2 y 3 muestran el movimiento de los músculos biológico a través de la fotopletismografía y electromiografía, respectivamente. Aunque las ondas no son iguales se diferencian en ambas la fricción y contracción del músculo. Cabe destacar que con el sensor de pulso solamente es necesario un punto de contacto; pero con el myosensor es necesario tres puntos de contacto, por lo tanto, requiere más área de piel. Por lo antes mencionado se puede considerar que los sensores de pulso son una alternativa compacta y económica a los myosensores.

La claridad de la onda de los sensores de pulso depende considerablemente del color de la piel del usuario y del grosor del tejido adiposo, además, los puntos del contacto del sensor, pueden variar de usuario en usuario, es por esto por lo que es necesario que para cada usuario a cuál se le vaya a aplicar este tipo de sensores se realice una etapa de calibración.

A pesar de que en este artículo no se logra construir un artículo funcional de una prótesis, ofrece datos experimentales $\mathrm{y}$ un estado del arte suficiente como para un trabajo futuro llegar a construir un prototipo de la prótesis.

Por los datos mostrados en la tabla 1 se distingue que, a menor calibre del alambre de cobre, menor será el voltaje necesario para producir el efecto Joule debido a que la resistencia es menor.

El modelo matemático que se obtiene en la sección de resultados sintetiza los datos experimentales y a su vez permite facilitar el control de los músculos artificiales por programación.

\section{REFERENCIAS}

[1] T. Fukushima, K. Asaka, A. Kosaka, and T. Aida, "Fully Plastic Actuator through Layer-by-Layer Casting with Ionic-LiquidBased Bucky Gel," Angew. Chemie, vol. 117, no. 16, pp. 24622465, Apr. 2005.

[2] R. E. Pelrine, R. D. Kornbluh, and J. P. Joseph, "Electrostriction of polymer dielectrics with compliant electrodes as a means of actuation," Sensors Actuators A Phys., vol. 64, no. 1, pp. 7785, Jan. 1998.

[3] K. Kruusamäe, A. Punning, A. Aabloo, and K. Asaka, "SelfSensing Ionic Polymer Actuators: A Review," Actuators, vol. 4 , pp. 17-38, 2015.

[4] K. Asaka, K. Oguro, Y. Nishimura, M. Mizuhata, and H. 
Takenaka, "Bending of Polyelectrolyte Membrane-Platinum Composites by Electric Stimuli I. Response Characteristics to Various Waveforms," Polym. J., vol. 27, no. 4, pp. 436-440, Apr. 1995.

[5] C. S. Haines et al., "Artificial Muscles from Fishing Line and Sewing Thread," Science (80-. )., vol. 343, no. 6173, pp. 868872, Feb. 2014.

[6] S. M. Mirvakili et al., "Simple and strong: twisted silver painted nylon artificial muscle actuated by Joule heating," 2014, vol. 9056, p. 90560I.

[7] A. N. Semochkin, "A device for producing artificial muscles from nylon fishing line with a heater wire," in 2016 IEEE International Symposium on Assembly and Manufacturing (ISAM), 2016, pp. 26-30.

[8] E. Jonsson et al., "Nylon-Muscle-Actuated Robotic Finger Citation: Nylon-Muscle-Actuated Robotic Finger," 2015.

[9] L. Wu, M. Jung de Andrade, L. K. Saharan, R. S. Rome, R. H. Baughman, and Y. Tadesse, "Compact and low-cost humanoid hand powered by nylon artificial muscles," Bioinspir. Biomim., vol. 12, no. 2, p. 026004, Feb. 2017.

[10] L. Saharan, M. J. de Andrade, W. Saleem, R. H. Baughman, and Y. Tadesse, "iGrab: hand orthosis powered by twisted and coiled polymer muscles," Smart Mater. Struct., vol. 26, no. 10, p. 105048 , Oct. 2017.

[11] M. Hiraoka et al., "Power-efficient low-temperature woven coiled fibre actuator for wearable applications," Sci. Rep., vol. 6, no. 1, p. 36358 , Dec. 2016.

[12] K. J. Kim, X. Tan, H. R. Choi, and D. Pugal, Biomimetic Robotic Artificial Muscles. World Scientific, 2013.
[13] Y. Tadesse, L. Wu, F. Karami, and A. Hamidi, "Biorobotic systems design and development using TCP muscles," in Electroactive Polymer Actuators and Devices (EAPAD) XX, 2018, vol. 10594, p. 40.

[14] H. H. Muhammed and J. Raghavendra, "Optomyography (OMG): A Novel Technique for the Detection of Muscle Surface Displacement Using Photoelectric Sensors."

[15] G. C. Righini, A. Tajani, and A. Cutolo, An introduction to optoelectronic sensors. World Scientific, 2009.

[16] A. Cherubini, G. Moretti, R. Vertechy, and M. Fontana, "Experimental characterization of thermally-activated artificial muscles based on coiled nylon fishing lines," AIP Adv., vol. 5, no. 6 , p. 067158 , Jun. 2015.

[17] C. L. Choy, F. C. Chen, and E. L. Ong, "Anistropic thermal expansion of oriented crystalline polymers," Polymer (Guildf)., vol. 20, no. 10, pp. 1191-1198, Oct. 1979.

[18] R. Online, C. S. Haines, N. Li, G. M. Spinks, A. E. Aliev, and J. Di, "New twist on artificial muscles," 2016.

[19] A. Love, "A treatise on the mathematical theory of elasticity."

[20] M. C. Yip and G. Niemeyer, "On the Control and Properties of Supercoiled Polymer Artificial Muscles," IEEE Trans. Robot., vol. 33, no. 3, pp. 689-699, Jun. 2017.

[21] M. Suzuki, N. Kamamichi, M. Suzuki, and N. Kamamichi, "Simple Controller Design Based on Internal Model Control for Twisted and Coiled Polymer Actuator," Actuators, vol. 7, no. 3, p. 33, Jun. 2018. 